\title{
SIMULAÇÃO E ANÁLISE TÉCNICO-ECONÔMICA DE UMA UNIDADE DE PRODUÇÃO DE METANOL A PARTIR DA GASEIFICAÇÃO DE BIOMASSA
}

\author{
K. Y. O. ALTOÉ ${ }^{1}$, F. A. RODRIGUES ${ }^{1}$
}

\author{
${ }^{1}$ Universidade Federal de Viçosa, Departamento de Química \\ E-mail para contato: kinaltoe@hotmail.com, fabio.rodrigues@ufv.br
}

\begin{abstract}
RESUMO- Neste trabalho foram avaliadas as etapas do ciclo de vida de uma planta de produção de metanol a partir da gaseificação do bagaço de cana. A síntese de rotas químicas, desenvolvimento de processos (modelagem termodinâmica e cinética) e engenharia de processos (definição dos equipamentos básicos para a avaliação econômica) foram investigados. Metanol foi escolhido devido às suas inúmeras utilizações. Assim, a gaseificação do bagaço de cana foi um estudo de caso simulado no software livre de processos químicos COCO. Considerando-se um consumo diário de 100 toneladas de bagaço de cana e um preço de venda de metanol U\$ \$2.00/kg, os resultados obtidos foram: produção anual de metanol de 13.600 toneladas, capital total investido de 39,269 milhões dólares; saldo líquido de venda U\$ \$ 27,21 milhões / ano, tempo de retorno de 2,9 anos; retorno de investimento de 20,6\%/ano. Para essa condição a planta é viável economicamente, como referência o mercado com um retorno de investimento de $15 \% /$ ano e tempo de retorno de 3,6 anos.
\end{abstract}

\section{INTRODUÇÃO}

Simuladores de processos tornaram-se ferramentas necessárias aos engenheiros de design, processos e aos operadores de planta. Quando usado adequadamente, o simulador pode ajudar o engenheiro a tornar-se mais produtivo e criativo na realização de suas tarefas (BROWN et al., 2006). O completo desenvolvimento do design de uma planta envolve a consideração de vários tópicos diferentes. Compreensivelmente, o quadro econômico global define se a instalação em questão será aprovada (PETERS e TIMMERHAUS, 2003).

A biomassa vem se afirmando como uma fonte de energia e matéria-prima, e exibe potencial para complementar ou mesmo substituir os materiais fósseis e seus derivados. As tecnologias de gaseificação não são recentes, mas mostram-se capazes de suprir as demandas energéticas mundiais, de maneira sustentável e ambientalmente correta, em um futuro não distante (SCHLITTLER et al., 2009). Assim, o objetivo deste trabalho foi a simulação no software livre COCO e a análise técnico-econômica, usando como caso de estudo, uma instalação industrial destinada à produção de metanol a partir da gaseificação de biomassa.

\section{MATERIAIS E MÉTODOS}

Segundo Lora e Venturini (2012 apud CIFRE e BADR, 2007), o processo de produção de metanol compreende as seguintes etapas: pré-tratamento de matérias-primas, gaseificação 
de biomassa, tratamento de gás de síntese, reforma de hidrocarbonetos, ajuste da razão $\mathrm{H}_{2} / \mathrm{CO}$, síntese de metanol e destilação. Neste trabalho apenas gaseificação da biomassa, síntese de metanol e destilação foram considerados. As seguintes hipóteses foram feitas a em relação ao processo de gaseificação: processo isotérmico em estado estacionário e sem perda de carga; biomassa composta apenas por carbono e livre de água; produtos de gaseificação se comportam como gases ideais: $\mathrm{CO}, \mathrm{CO}_{2}, \mathrm{H}_{2}$ e $\mathrm{CH}_{4}$; reações de gaseificação atingem $\mathrm{o}$ equilíbrio.

Para modelar o processo de gaseificação usou-se o modelo de reator de Gibbs e as condições de entrada assumidas por Tan e Zhong (2010): biomassa a 101,3 kPa e $288 \mathrm{~K}$, vapor a $101,3 \mathrm{kPa}$ e $673 \mathrm{~K}$, gaseificador operando a 101,3 kPa e $973 \mathrm{~K}$ e uma razão molar vapor/biomassa alimentada ao gaseificador igual a 1. Devido a não-existência de carbono como um elemento simples na base de dados do $\mathrm{COCO}^{\circledR}$ usou-se o software UniSim R410 ${ }^{\circledR}$ para simular o processo de gaseificação. Os dados obtidos foram inseridos ao simulador de processos químicos $\mathrm{COCO}^{\circledR}$.

Para o reator de metanol usou-se os dados fornecidos por Panahi et al. (2012): catalisador com massa específica de $1063 \mathrm{~kg} / \mathrm{m}^{3}$ e diâmetro de partícula de $0,04 \mathrm{~m}$. De acordo com o trabalho de Yusup et al. (2010), observou-se uma grande discrepância entre o valor do diâmetro da partícula $(0,006 \mathrm{~m})$ e o utilizado $(0,04 \mathrm{~m})$. De modo a adaptar-se o último foi utilizado o valor de $0,004 \mathrm{~m}$. Assim, utilizou-se um valor de porosidade do leito de 0,5 .

Para a reação de metanol o modelo desenvolvido por Vanden Busschee Froment (1996) foi considerado, de acordo com as Reações 1 e 2 e suas respectivas taxas dadas pelas equações 1 e 2 :

$$
\begin{aligned}
& \mathrm{CO}_{2}+3 \mathrm{H}_{2} \leftrightarrow \mathrm{CH}_{3} \mathrm{OH}+\mathrm{H}_{2} \mathrm{O} \\
& \mathrm{CO}_{2}+\mathrm{H}_{2} \leftrightarrow \mathrm{CO}+\mathrm{H}_{2} \mathrm{O}
\end{aligned}
$$

O pacote termodinâmico Peng Robinson foi utilizado para realizar as simulações.

Os gás de síntese obtido é comprimido até $5100 \mathrm{kPa}$ (pressão do reator) passando por estágios intermediários de resfriamento. Adotou-se uma temperatura de saída de cada etapa de resfriamento de $373 \mathrm{~K}$, uma relação de compressão igual a 3 e uma queda de pressão de 20 $\mathrm{kPa}$ para cada etapa de resfriamento, de acordo com Turton et al. (2009). Como praticamente todo $\mathrm{H}_{2}$ foi consumido nas reações de formação de metanol não se verificou a necessidade de reciclo. O efluente do reator passa por um resfriador atingindo $353 \mathrm{~K}$. Como há formação de fase líquida, uma queda de pressão de $10 \mathrm{kPa}$ foi adotada na sequência de acordo com Turton et al. (2009). Esta corrente passa através de uma válvula, igualando sua pressão à atmosférica e atingindo um vaso de flash onde o metanol é separado.

Na Figura 1 encontra-se o diagrama de fluxo do processo desenvolvido no software livre COCO: 


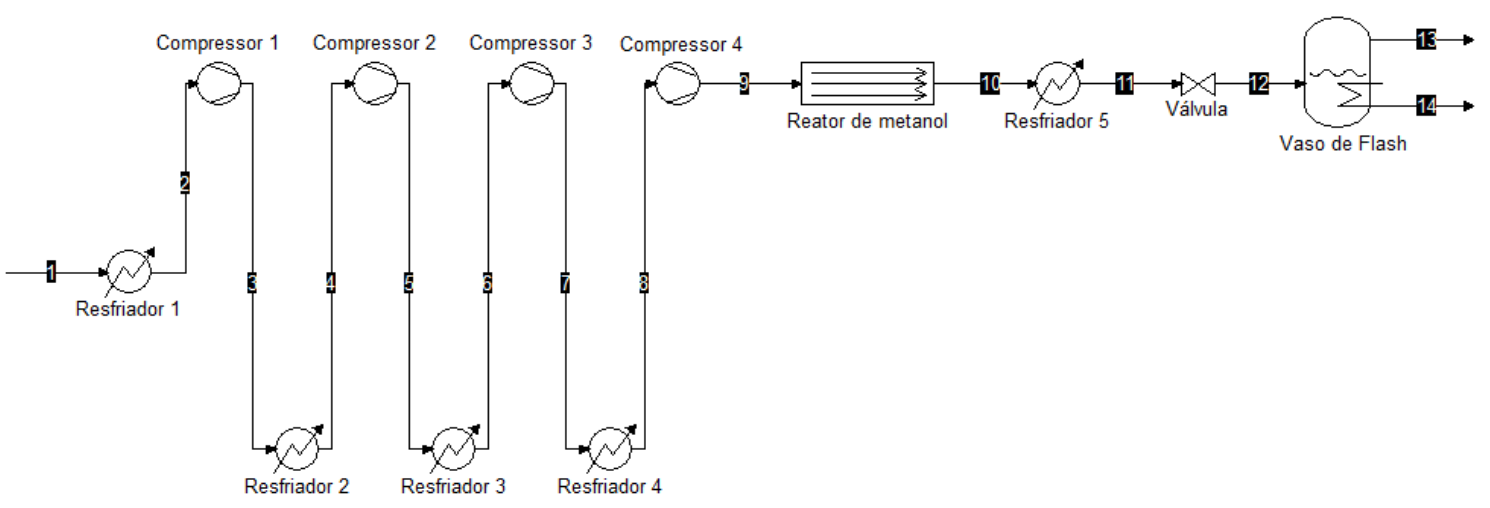

Figura 1 - Diagrama de fluxo do processo de produção do metanol.

O procedimento adotado para fazer a análise econômica foi realizado de acordo com a planilha em EXCEL proposta por Peters e Timmerhaus (2003) e é descrito a seguir: Estima-se o custo de compra de cada equipamento do processo, Na planilha "Capital Inv" entra-se com o custo total de compra dos equipamentos do processo para calcular o investimento de capital fixo e capital de giro, Na planilha "Material \& Labor" entra-se com o preço de produtos e matérias-primas com suas respectivas vazões além da mão-de-obra requerida, Na planilha "Utilities" entra-se com as quantidades de utilidades anuais necessárias com as unidades apropriadas, A planilha "Anual TOTAL PRODUCT COST" calcula-se o custo total anual do produto e na planilha "Evaluation" estabelece-se o fluxo de caixa e calcula as medidas de rentabilidade comum, tais como, tempo de pagamento, retorno do investimento e retorno líquido.

O custo de compra dos equipamentos pode ser estimado pela Equação 3, considerando pressão de 1 atm e de aço de carbono como material de construção:

$$
\log \left(C_{p}^{0}\right)=K_{1}+K_{2} \log (A)+K_{3}(\log (A))^{2}
$$

sendo A o tamanho ou capacidade do equipamento e os parâmetros $K_{1}, K_{2}$ e $K_{3}$ suas constantes características, podendo ser encontrados no trabalho de Turton et al. (2009).

O fator de material $\left(\mathrm{F}_{\mathrm{M}}\right)$ é responsável pelo ajuste do custo do equipamento referente ao seu material de construção enquanto o fator de condição $\left(\mathrm{F}_{\mathrm{P}}\right)$ ajusta o custo do equipamento para as condições de operação de temperatura e pressão. A partir dos valores de $C_{p}^{0}, \mathrm{~F}_{\mathrm{P}}$ e $\mathrm{F}_{\mathrm{M}}$ pode-se calcular o custo bare module $\left(\mathrm{C}_{\mathrm{BM}}\right)$, através da Equação 4:

$$
C_{B M}=C_{p}^{0} F_{B M}=C_{p}^{0}\left(B_{1}+B_{2} F_{M} F_{p}\right)
$$

sendo $\mathrm{F}_{\mathrm{BM}} \mathrm{O}$ fator bare module e as constantes $\mathrm{B}_{1}$ e $\mathrm{B}_{2}$ específicas de cada tipo de equipamento, de acordo com Turton et al. (2009). Ajusta-se o valor do custo de capital no tempo mediante índices específicos da indústria de acordo com a Equação 5: 


$$
C_{2}=C_{1}\left(\frac{I_{2}}{I_{1}}\right)
$$

onde $\mathrm{C}_{1}$ e $\mathrm{I}_{1}$ representam, respectivamente, o custo e o índice de inflação do ano base e $\mathrm{C}_{2}$ e $\mathrm{I}_{2}$ do ano em questão. Neste trabalho foi usado o índice CEPCI (Chemical Engineering Plant Cost Index), considerando-se o ano-base de 2001 (397) e no ano em questão de 2012 (572,7).

\section{RESULTADOS}

Os dados obtidos foram comparados com os dados publicados por Tan e Zhong (2012) e encontram-se na Tabela 1:

Tabela 1 - Composição do gás de síntese em base seca (\% vol)

\begin{tabular}{|c|c|c|c|c|}
\hline Componente & $\mathrm{H}_{2}$ & $\mathrm{CO}$ & $\mathrm{CO}_{2}$ & $\mathrm{CH}_{4}$ \\
\hline Tan e Zhong (2012) & 61,17 & 18,86 & 19,42 & 0,49 \\
\hline Simulação & 45,37 & 48,68 & 2,15 & 3,8 \\
\hline
\end{tabular}

As discrepâncias entre os valores podem ser explicados pelo modelo de reator de Gibbs com as reações pré-definidas utilizadas por Tan e Zhong (2012), dispostas na Tabela 2, e pela composição mássica da biomassa adotada pelos autores: 45,76 \% carbono, 6,74 \% hidrogênio, $0,07 \%$ nitrogênio e $47,42 \%$ oxigênio.

Tabela 2 - Reações de gaseificação

\begin{tabular}{|l|l|}
\hline $3 \mathrm{C}+2 \mathrm{O}_{2} \rightarrow 2 \mathrm{CO}+\mathrm{CO}_{2}$ & (6) \\
\hline $\mathrm{C}+\mathrm{H}_{2} \mathrm{O} \rightarrow \mathrm{CO}+\mathrm{H}_{2}$ & $(7)$ \\
\hline $\mathrm{CO}+\mathrm{H}_{2} \mathrm{O} \rightarrow \mathrm{CO}_{2}+\mathrm{H}_{2}$ & $(2)$ \\
\hline $\mathrm{C}+\mathrm{CO}_{2} \rightarrow 2 \mathrm{CO}$ & $(8)$ \\
\hline $\mathrm{C}+2 \mathrm{H}_{2} \rightarrow \mathrm{CH}_{4}$ & $(9)$ \\
\hline $\mathrm{CH}+\mathrm{H}_{2} \mathrm{O} \rightarrow \mathrm{CO}+3 \mathrm{H}_{2}$ & (10) \\
\hline $\mathrm{CH}_{4}+2 \mathrm{H}_{2} \mathrm{O} \rightarrow \mathrm{CO}+4 \mathrm{H}_{2}$ & $(11)$ \\
\hline
\end{tabular}

Os dados obtidos para a reação de metanol foram comparados com os dados publicados por Panahi et al. (2012). Avaliou-se o rendimento de metanol em relação ao conteúdo de hidrogênio e carbono de acordo com as seguintes equações:

$$
\begin{aligned}
& Y_{\mathrm{CH}_{\mathrm{g}} \mathrm{OH}} / \mathrm{H}_{\mathrm{z}}=\frac{F_{\mathrm{CH}_{\mathrm{g}} \mathrm{OH}}}{F_{\mathrm{H}_{2}}^{0}} \times 100 \\
& Y_{\mathrm{CH}_{\mathrm{g}} \mathrm{OH} / \mathrm{CO}+\mathrm{CO}_{2}}=\frac{F_{\mathrm{CH}_{\mathrm{g}} \mathrm{OH}}}{F_{\mathrm{CO}}^{0}+F_{\mathrm{CO}_{2}}^{0}} \times 100
\end{aligned}
$$


onde $F_{\mathrm{CH}_{\mathrm{g}} \mathrm{OH}}$ é o fluxo molar de metanol na saída do reator e $F_{\mathrm{H}_{2}}^{0}$, $F_{\mathrm{CO}}^{0}$ e $F_{\mathrm{CO}_{2}}^{0}$ são, respectivamente, os fluxos molares de entrada de hidrogênio, monóxido e dióxido de carbono.

A Equação 12 mostra a percentagem de hidrogênio convertido em metanol enquanto a Equação 13 a percentagem de carbono convertido em metanol. Os valores obtidos a partir das equações 12 e 13 por Panahi et al. (2012) foram, respectivamente, 7,052 e 73,17. A simulação obteve 51,82 e 46,26 , respectivamente.

Para entender melhor os resultados obtidos, as composições molares de entrada e as condições reacionais foram analisadas e encontram-se na Tabela 3 :

Tabela 3 - Dados reacionais

\begin{tabular}{|c|c|c|c|c|c|c|}
\hline \multirow{2}{*}{$\begin{array}{l}\text { Panahi et al. } \\
\text { (2012) }\end{array}$} & $\mathrm{T}_{\text {ent }}$ & $498 \mathrm{~K}$ & $P_{\text {ent }}$ & $8200 \mathrm{kPa}$ & \multirow{2}{*}{$\begin{array}{l}\text { Composição } \\
\text { (\% mol) }\end{array}$} & \multirow{2}{*}{$\begin{array}{c}80 \mathrm{H}_{2} ; 4,76 \mathrm{CO} ; 2,95 \mathrm{CO}_{2} \\
\quad 11,92 \mathrm{CH}_{4} ; 0,06 \mathrm{H}_{2} \mathrm{O}\end{array}$} \\
\hline & $\mathrm{T}_{\text {sai }}$ & $528 \mathrm{~K}$ & $\mathrm{P}_{\text {sai }}$ & $8200 \mathrm{kPa}$ & & \\
\hline \multirow{2}{*}{ Simulação } & $\mathrm{T}_{\text {ent }}$ & $524,96 \mathrm{~K}$ & $P_{\text {ent }}$ & $5100 \mathrm{kPa}$ & \multirow{2}{*}{$\begin{array}{l}\text { Composição } \\
\text { (\% mol })\end{array}$} & \multirow{2}{*}{$\begin{array}{l}44,64 \mathrm{H}_{2} ; 47,89 \mathrm{CO} ; 2,11 \\
\mathrm{CO}_{2} ; 3,74 \mathrm{CH}_{4} ; 1,62 \mathrm{H}_{2} \mathrm{O}\end{array}$} \\
\hline & $\mathrm{T}_{\text {sai }}$ & $524,96 \mathrm{~K}$ & $\mathrm{P}_{\text {sai }}$ & $5098 \mathrm{kPa}$ & & \\
\hline
\end{tabular}

Em relação ao trabalho de Panahi et al. (2012), menores valores de rendimento de metanol em comparação com o teor de hidrogênio e valores mais elevados em comparação com o teor de carbono podem ser explicados pela alta pressão, elevado teor de hidrogênio e baixo teor de $\mathrm{CO}$ e $\mathrm{CO}_{2}$.

A simulação apresenta um baixo conteúdo de $\mathrm{H}_{2}$ e $\mathrm{CO}_{2}$ e um alto teor de $\mathrm{CO}$. Por conseguinte, um elevado rendimento de metanol em relação ao teor de hidrogênio e um baixo rendimento em relação ao teor de carbono são explicados.

Para efetuar-se o cálculo de compra dos equipamentos, os mesmos foram modelados da seguinte forma: gaseificador como aquecedor de água quente, os compressores como do tipo rotatório sendo aço-carbono seu material de construção, o reator foi como encamisado agitado com $3 \mathrm{~m}$ de comprimento e $1 \mathrm{~m}$ diâmetro (estas dimensões foram adotadas apenas para executar a simulação).

O vaso de flash foi modelado de acordo com Turton et al. (2009).

Para o vaso de flash foram obtidos um diâmetro de 1,255 m e comprimento de 0,8166 m. Sua configuração foi considerada vertical e aço inoxidável seu material de construção. Para os resfriadores, um fluxo mássico de água de $63,4916 \mathrm{~kg} / \mathrm{s}$ foi calculado considerando um aumento de temperatura de $15^{\circ} \mathrm{C}$ e um calor específico de $4,2 \mathrm{~kJ} / \mathrm{kg}^{\circ} \mathrm{C}$.

Considerando todos os dados de dimensionamento apresentados anteriormente calculou-se o custo de aquisição dos equipamentos: US\$ 4.172.830,59 para o ano de 2001. 
Corrigindo esse valor para o ano de 2012 de acordo com a Equação 7, um valor de US\$ 6.019.597,18 foi obtido.

A partir dos dados de custo dos equipamentos, considerando que biomassa e vapor foram obtidos sem custos, o consumo anual de água de resfriamento foi de $1.919 .986 \mathrm{~m}^{3}$, o preço de venda do metanol de US\$2,00/kg, o ciclo de vida da planta de 10 anos e 350 dias/ano de operação utilizou-se a planilha desenvolvida por Peters e Timmerhaus (2003), para calcular os dados econômicos. Estes podem ser encontrados na Tabela 4:

Tabela 4 - Dados econômicos

\begin{tabular}{|c|c|c|c|}
\hline $\begin{array}{c}\text { Consumo de biomassa } \\
\text { (ton/dia) }\end{array}$ & 100 & $\begin{array}{c}\text { Capital total investido } \\
\text { (milhões US\$) }\end{array}$ & 39,269 \\
\hline $\begin{array}{c}\text { Produção de metanol } \\
\text { (ton/ano) }\end{array}$ & 13.600 & $\begin{array}{c}\text { Lucro líquido (milhões } \\
\text { US\$/ano) }\end{array}$ & 2,28 \\
\hline $\begin{array}{c}\text { Capital fixo investido } \\
\text { (milhões US\$) }\end{array}$ & 33,375 & $\begin{array}{c}\text { Tempo de retorno } \\
\text { (anos) }\end{array}$ & 2,9 \\
\hline $\begin{array}{c}\text { Capital de giro } \\
\text { (milhões US\$) }\end{array}$ & 5,894 & $\begin{array}{c}\text { Retorno sobre } \\
\text { investimento }(\%)\end{array}$ & 20,6 \\
\hline
\end{tabular}

Assim sendo, o investimento na construção de tal projeto é rentável uma vez que o retorno do investimento obtido foi de $20,6 \%$ e o tempo de retorno de 2,9 anos, superior aos $15 \%$ e 3,6 anos recomendado por Peters e Timmerhaus (2003), respectivamente.

\section{CONCLUSÃO}

A partir dos dados financeiros obtidos pode-se concluir que a implementação do projeto considerado neste trabalho tem viabilidade econômica uma vez que o retorno do investimento obtido foi de $20,6 \%$ e o tempo de retorno igual a 2,9 anos. Tais perspectivas devem, no entanto, ser relativizadas devido às suposições efetuadas (preço de venda do metanol produzido e o custo das matérias-primas) e às limitações técnicas do simulador de processos químicos $\mathrm{COCO}^{\circledR}$ (banco de dados limitado e algoritmos menos robustos, por exemplo), o que tende a restringir o grau de aproximação entre os dados de simulação e os do processo real.

\section{AGRADECIMENTO}

Os autores agradecem a empresa HONEYWELL pelo fornecimento do software UNISIM $^{\mathrm{TM}}$ e a FAPEMIG pelo apoio financeiro. 


\section{NOMENCLATURA}

\begin{tabular}{|c|l|c|}
\hline $\mathrm{A}$ & Parâmetro de capacidade/tamanho & - \\
\hline $\mathrm{B}_{1}, \mathrm{~B}_{2}$ & Constante do fator bare module & - \\
\hline $\mathrm{D}$ & Diâmetro & $\mathrm{m}$ \\
\hline $\mathrm{F}$ & Fluxo molar & $\mathrm{mol} / \mathrm{s}$ \\
\hline $\mathrm{F}_{\mathrm{M}}$ & Fator de material & - \\
\hline $\mathrm{F}_{\mathrm{P}}$ & Fator de pressão & - \\
\hline $\mathrm{f}_{\mathrm{S}}$ & Fator de segurança & - \\
\hline $\mathrm{I}$ & Indice de inflação & - \\
\hline $\mathrm{K}_{1}, \mathrm{~K}_{2}, \mathrm{~K}_{3}$ & Parâmetro de capacidade/tamanho & - \\
\hline $\mathrm{k}$ & Coeficiente de velocidade máxima & $\mathrm{m} / \mathrm{s}$ \\
\hline $\mathrm{L}$ & Comprimento & $\mathrm{m}$ \\
\hline $\mathrm{P}$ & Pressão & $\mathrm{kPa}$ \\
\hline $\mathrm{T}$ & Temperatura & $\mathrm{K}$ \\
\hline $\mathrm{V}$ & Volume específico molar & $\mathrm{m}^{3} / \mathrm{mol}$ \\
\hline $\mathrm{V}$ & Volume & $\mathrm{m}$ \\
\hline $\mathrm{Y}$ & Rendimento de metanol em relação a um componente & - \\
\hline $\mathrm{t}_{\mathrm{L}}$ & Tempo de residência do líquido & $\mathrm{s}$ \\
\hline$\rho$ & Massa específica & $\mathrm{kg} / \mathrm{m}^{3}$ \\
\hline
\end{tabular}

\section{REFERÊNCIAS}

BROWN, W. G.; OCHS, L.; VARNER, J. W. Using process simulators will make your plant more productive and efficient. Gas Processors Association, 2006.

CIFRE, P. G.; BADR, O. Renewable hydrogen utilization for the production of methanol. En. Conv. and Mgmt, v. 48, n. 2, p. 519-527, 2007.

LORA, E. E. S.; VENTURINI, O. J. Biocombustíveis. 1. ed. Rio de Janeiro: Interciência, 2012. v. 1.

PANAHI, P. N.; MOUSAVI, S. M.; NIAEI, A.; FARZI, A.; SALARI, D. Simulation of methanol synthesis from synthesis gas in fixed bed catalytic reactor using mathematical modeling and neural networks. IJSER, v. 3, n. 2, 2012.

PETERS, M. S.; TIMMERHAUS, K. D. Plant design and economics for chemical engineers. 5 ed. New York: McGraw-Hill, 2003.

SCHlitTler, L. A. F. S.; GOMES, E. B.; ANTUNES, A. M. S.; JÚNIOR, N. P. Gaseificação de biomassa. Diálogos \& Ciência, n. 11, 2009.

TAN, W.; ZHONG, Q. Simulation of hydrogen production in biomass gasifier by ASPEN PLUS. In: Power and Energy Engineering Conference (APPEEC), 2010 Asia-Pacific, 2, 2010, China. 
TURTON, R.; BAILIE, R. C.; WHITING, W. B.; SHAEIWITZ, J. A. Analysis, synthesis, and design of chemical processes. 3. ed. New York: Prentice Hall, 2009. 1068 p.

UNISim HONEYWELL， <www.honeywell.com>

VANDEN BUSSCHE, K. M.; FROMENT, G. F. A Steady-state kinetic model for methanol synthesis and the water gas shift reaction on a commercial $\mathrm{Cu} / \mathrm{ZnO} / \mathrm{Al} 2 \mathrm{O} 3$ catalyst. J. of Catal., n. 161, p. 1-10, 1996.

YUSUP, S.; ANH, N. P.; ZABIRI, H. A simulation study of an industrial methanol reactor based on simplified steady state methanol. IJRRAS, v. 5, n. 3, 2010. 